\title{
Effects of Gait Training With Body Weight Support on a Treadmill Versus Overground in Individuals With Stroke
}

\author{
Gabriela L. Gama, MSc, ${ }^{a}$ Melissa L. Celestino, MSc, ${ }^{a}$ José A. Barela, PhD, ${ }^{a, b}$ \\ Larry Forrester, $\mathrm{PhD},{ }^{\mathrm{c}}$ Jill Whitall, $\mathrm{PhD},{ }^{\mathrm{d}, \mathrm{e}}$ Ana M. Barela, $\mathrm{PhD}^{\mathrm{a}}$
}

From the ${ }^{a}$ Institute of Physical Activity and Sport Sciences, Cruzeiro do Sul University, São Paulo, SP; ${ }^{b}$ Department of Physical Education, São Paulo State University, Rio Claro, SP, Brazil; ' $M a r y l a n d$ Exercise \& Robotics Center of Excellence, Veterans Administration Maryland Health Care System, Baltimore, MD; ${ }^{d}$ Department of Physical Therapy and Rehabilitation Science, School of Medicine, University of Maryland, Baltimore, MD; and ${ }^{e}$ Faculty of Health Sciences, University of Southampton, Southampton, United Kingdom.

\begin{abstract}
Objective: To investigate the effects of gait training with body weight support (BWS) on a treadmill versus overground in individuals with chronic stroke.

Design: Randomized controlled trial.

Setting: University research laboratory.

Participants: Individuals $(\mathrm{N}=28)$ with chronic stroke ( $>6 \mathrm{mo}$ from the stroke event).

Interventions: Participants were randomly assigned to receive gait training with BWS on a treadmill $(\mathrm{n}=14)$ or overground $(\mathrm{n}=14) 3$ times a week for 6 weeks.

Main Outcome Measures: Gait speed measured using the 10-meter walk test, endurance measured using the 6-minute walk test, functional independence measured using the motor domain of the FIM, lower limb recovery measured using the lower extremity domain of the Fugl-Meyer assessment, step length, step length symmetry ratio, and single-limb support duration. Measurements were obtained at baseline, immediately after the training session, and 6 weeks after the training session.

Results: At 1 week after the last training session, both groups improved in all outcome measures except paretic step length and step length symmetry ratio, which were improved only in the overground group $(P=.01$ and $P=.01$, respectively). At 6 weeks after the last training session, all improvements remained and the treadmill group also improved paretic step length $(P<.001)$ but not step length symmetry ratio $(P>.05)$. Conclusions: Individuals with chronic stroke equally improve gait speed and other gait parameters after 18 sessions of BWS gait training on either a treadmill or overground. Only the overground group improved step length symmetry ratio, suggesting a role of integrating overground walking into BWS interventions poststroke.
\end{abstract}

Archives of Physical Medicine and Rehabilitation 2017;98:738-45

(c) 2016 by the American Congress of Rehabilitation Medicine

Partial body weight support (BWS) systems have been widely used for gait rehabilitation poststroke. ${ }^{1-7}$ In individuals with stroke, these systems improved body weight distribution between paretic and nonparetic limbs as erect posture is facilitated, ${ }^{5}$ and they promote improvement in spatial-temporal gait characteristics,

Supported by the São Paulo Research Foundation - FAPESP and by fellowship from Coordenação de Aperfeiçoamento de Pessoal de Nível Superior - CAPES. All financial support had no influence on analysis and interpretation of data or writing of manuscript.

Clinical Trial Registration No.: NCT02088255.

Disclosures: none. including interlimb symmetry of stance and swing phases, muscle activity, and joint angle excursions of the lower limbs. ${ }^{8}$ BWS used with treadmills facilitates performance of a high number of symmetrical and consistent steps, ${ }^{9}$ as well as allowing control of walking speed. In contrast, the requirements for walking on treadmills are different from those for overground walking, mainly in terms of propulsion and balance. ${ }^{10,11}$

Treadmills may provide a degree of passive movement to the lower limbs with little change in muscular activation, ${ }^{8,9,12-14}$ limiting the extent of skill transfer to overground walking. This 
raises a question whether BWS alone promotes improvement in gait performance or whether this is due to the interaction between BWS and treadmill. A related question is whether using gait training with BWS on would yield any differences in gait improvement compared with BWS on the treadmill.

Only 2 studies have used overground BWS training in individuals with stroke, including a case study ${ }^{15}$ and a single-arm study with a small sample. ${ }^{16}$ Sousa et $\mathrm{al}^{16}$ reported improvements in walking speed and step length symmetry ratio as well as increased stride lengths and segmental angle rotations ${ }^{17}$ after overground BWS gait training, but the study lacked a treadmill comparison group. Comparing BWS gait training in a controlled treadmill versus overground experiment may reveal differential effects due to use of BWS on a moving versus stationary walking surface. The purpose of this study was to investigate the effects of moving versus stationary walking surfaces during BWS gait training on measures of spatial-temporal gait parameters and clinical function in individuals with chronic stroke. It was hypothesized that overground BWS training would elicit a greater improvement in walking performance than would treadmill BWS training, because the former would reduce the need for skill transfer from the externally driven treadmill to fully self-generated control of overground mobility tasks.

\section{Methods}

A randomized controlled trial was conducted using Consolidated Standards of Reporting Trials guidelines. The study was approved by the research ethics committee of Cruzeiro do Sul University and was registered at ClinicalTrials.gov (Clinical Trial Registration No.: NCT02088255). All procedures were performed with adequate understanding, and written, signed informed consent of the participants was obtained before entry into the study.

\section{Setting and participants}

From April 1, 2014, to June 15, 2015, 114 individuals with stroke were contacted and invited to join the study (fig 1). One physical therapist researcher screened all potential participants. Inclusion criteria were chronic hemiparetic gait after an ischemic or hemorrhagic stroke, $>6$ months from the stroke event, absence of cardiac (or medical clearance for participation), orthopedic, or pulmonary disease or other neurologic impairment that could compromise gait or training, ability to follow 2-step verbal commands, and ability to walk $10 \mathrm{~m}$ with or without assistance. Individuals who presented with uncontrolled blood pressure were excluded. All stages of the study were conducted in the Movement Analysis Laboratory at Cruzeiro do Sul University, São Paulo, São Paulo, Brazil.

\section{Randomization and blinding}

After baseline testing, and using a computer-based algorithm, participants were randomly allocated to 2 BWS groups: treadmill

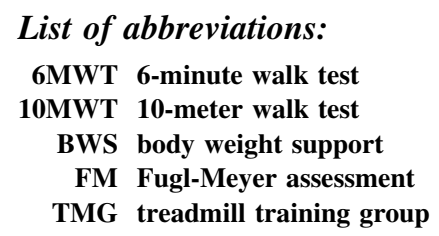

training group (TMG) or overground training group. The researcher who did the randomization and data analyses was not involved in any assessment or training session. Complete and continuous blinding of researchers who performed the training sessions and the assessments was not feasible because of personnel constraints. Participants were not blinded to the training conditions, but were unaware of the hypothesis of the study.

\section{Intervention protocols}

The BWS system used for the TMG consisted of a treadmill (model TK35 ${ }^{\mathrm{a}}$ ) and a metal frame with an instrumented load cell to register the amount of supported body weight. The BWS system (fig 2) used for the overground training group consisted of a suspended rail $(7 \mathrm{~m})$ mounted on the ceiling $(3 \mathrm{~m})$ and supported by 2 steel beams. ${ }^{\text {b }}$ A moving cart was attached to the bottom of the rail, allowing backward and forward movements and controlled by a belt system linked to a servomotor. A customized program $\left(\right.$ LabVIEW $^{\mathrm{c}}$ ) was developed to control displacement, velocity, and acceleration of the moving cart. In addition, a second servomotor controlled unloading of body weight through a harness and instrumented load cell system.

Training sessions were conducted 3 times weekly, with at least 1 day between sessions, for 6 weeks, totaling 18 training sessions. Each session lasted up to 45 minutes. Throughout the training sessions, each participant's heart rate was monitored and blood pressure was measured at the beginning and end of each session. Rest intervals were provided according to individual needs.

The amount of BWS during training sessions ranged from $30 \%$ to $0 \%$ of body weight. The amount of BWS was based on the individual alignment of trunk and limbs with proper weight shift and bearing onto the hemiplegic limb during the loading phases of gait. Walking speed was set to match participants' comfortable level on the treadmill or overground. The overground training group walked back and forth along the walkway. Participants were allowed to use the front handrail of the treadmill (TMG) or the therapist's hand (overground training group), but through training sessions, all participants were encouraged to walk without any external assistance other than the BWS system.

Progression of the training was achieved by reducing the BWS, increasing the gait speed, and/or reducing the patient support from the handrail for the TMG and the therapist's hand for the overground training group. The training parameter changes to progress the training were implemented at the beginning of each session. The progression was maintained as long as the participants could maintain alignment of trunk and limbs with proper weight shift and bearing onto the hemiplegic limb during the loading phases of gait. If not, the parameter change was decreased to the previous value. Two trained therapists conducted all training sessions of both groups and provided similar verbal and manual cues.

\section{Outcome measures and follow-up}

Participants were assessed 1 week before the first training session, 1 week after the last gait training session, and 6 weeks after the last training session. One experienced researcher took the lead on all assessments, and standardized instructions for each test were given to ensure consistency in test administration. Participants underwent the following evaluations: 10-meter walk test (10MWT), ${ }^{18-20}$ 6-minute walk test (6MWT),${ }^{21}$ motor domain of the FIM, ${ }^{22,23}$ lower extremity domain of the Fugl-Meyer 


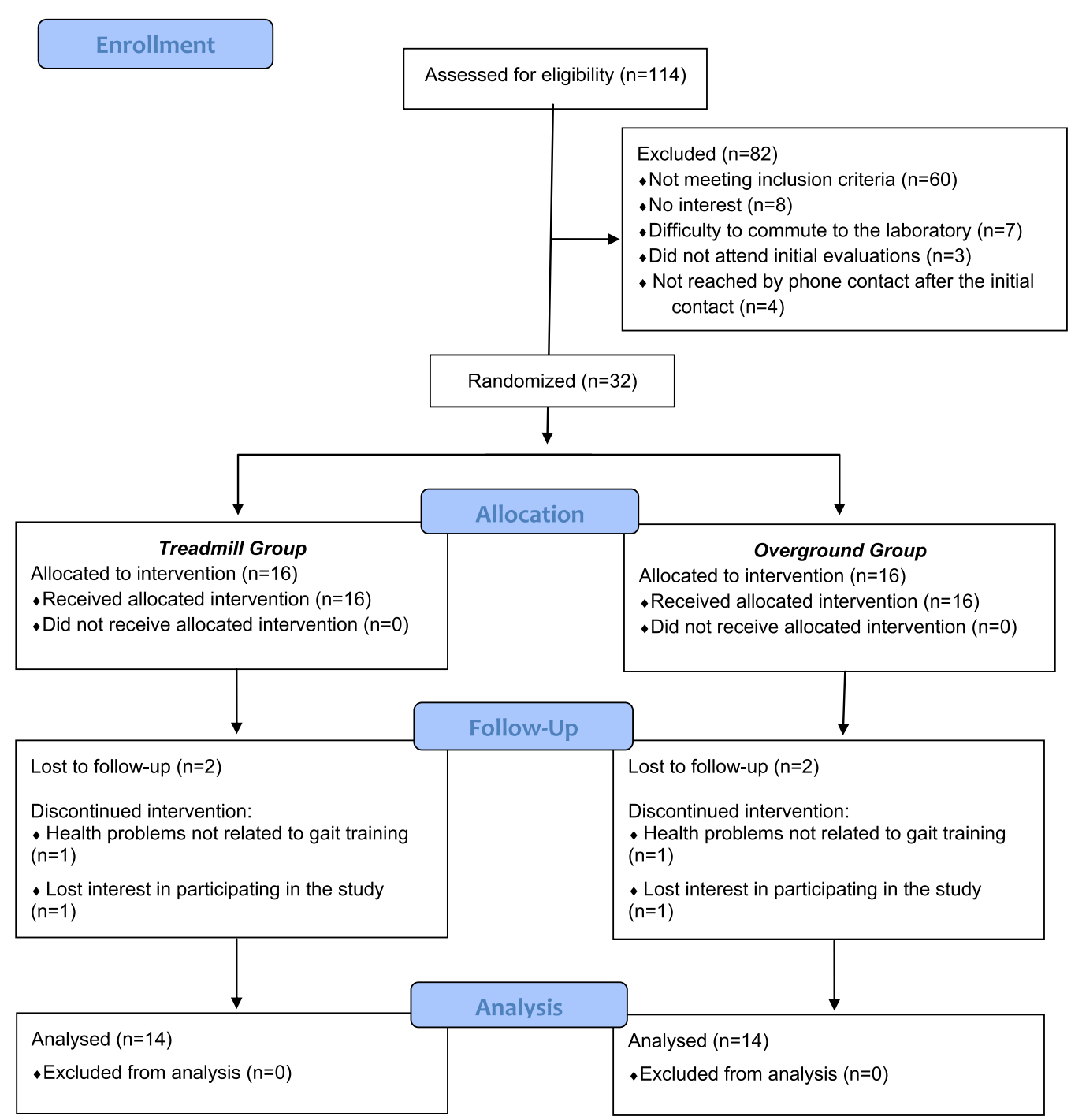

Fig 1 Flow diagram of the study following Consolidated Standards of Reporting Trials guidelines.

assessment (FM), ${ }^{24}$ and quantitative gait analyses (see below). Rest intervals were provided between tests according to individual needs, when necessary.

The primary outcome measure was gait speed measured using the 10MWT. During this test, participants were required to walk $10 \mathrm{~m}$ at a comfortable speed. Two photocells ${ }^{\mathrm{a}}$ measured the time

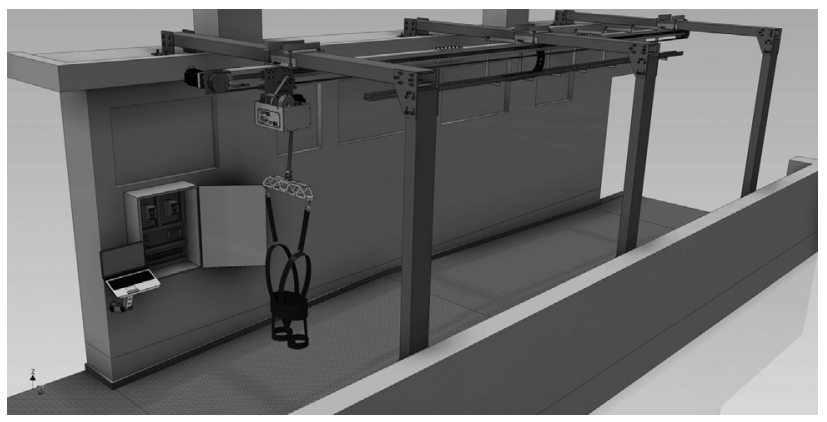

Fig 2 Representation of the BWS system adopted by the overground training group. required to cover the intermediate $6 \mathrm{~m}$ to exclude periods of acceleration and deceleration. The average of 3 trials was calculated.

Secondary outcome measures included endurance measured using the 6MWT, functional independence measured using the motor domain of the FIM, lower limb recovery measured using the lower extremity domain of the FM, which evaluated the subscales of joint pain, passive joint motion, sensibility, voluntary movement, reflex activity, and coordination. Finally, paretic and nonparetic step length, step length symmetry ratio, and paretic and nonparetic single-limb support were measured using a computerized gait analysis system during walking at a comfortable speed. For both 10MWT and 6MWT, participants were allowed to use assistive devices, if necessary.

The gait analysis was performed on a $7 \mathrm{~m}$ walkway equipped with 2 embedded force platforms (model 9286BA ${ }^{\mathrm{d}}$ ). A computerized gait analysis system $\left(\mathrm{VICON}^{\mathrm{e}}\right)$ with 7 infrared cameras (Bonita $10^{\mathrm{e}}$ ) was used to acquire data from reflective markers that were placed on the main body landmarks (Vicon Plug-In Gait model $\left.l^{\mathrm{e}}\right) .{ }^{25}$ After a calibration trial, participants were asked to walk at a comfortable self-selected speed. They were not allowed to use any assistive devices, but, when necessary, a therapist offered her 
hand to assist their balance without providing any meaningful mechanical support (see Results).

\section{Data processing of gait analysis}

Two consecutive and steady-state strides (paretic and nonparetic) (4 steps) per trial by each participant were analyzed for a total of 3 selected trials for each evaluation. A stride (walking cycle) was defined by 2 consecutive initial contacts of the same limb with the ground along the progression line. In addition, foot contacts and toe-offs during a stride were identified for subsequent calculation of the spatial-temporal organization of walking for both paretic and nonparetic sides.

The gait outcome measures analyzed in this study were step length, calculated as the distance between the initial contact of each foot along the progression line (determined by the position of the heel markers); step length symmetry ratio, calculated as the ratio between the shorter step and the longer step (we were interested in the magnitude of asymmetry rather than its direction) ${ }^{26}$; and single-limb support duration (determined by the time interval the limb supports total body weight while the contralateral limb swings). Data analysis was performed using a customized routine written in MATLAB. ${ }^{\mathrm{f}}$ Data from 3 trials for each evaluation were averaged for each participant and considered for further analyses.

\section{Statistical analysis}

For all outcome measures, comparisons between the following assessments were made: baseline test and 1 week after the last gait training session and baseline test and 6 weeks after the last training session. One participant from the overground training group missed assessment at 6 weeks after the last training session, and her scores from the 1 week after the last gait training session assessment were used to replace missing data, a conservative assumption. ${ }^{27,28}$ We used a schematic box plot for the primary outcome measurement to test for the existence of outliers, and we did not find any outlier. Two-way analysis of variance and multivariate analysis of variance were used using group (TMG and overground training group) and time of assessment (baseline test and 1 week after the last gait training session; baseline test and 6 weeks after the last training session) as factors. The dependent variables for the analyses of variance were 10MWT, 6MWT, FIM, FM, and step length symmetry ratio. The dependent variables for the multivariate analyses of variance were step length and singlelimb support of paretic and nonparetic sides. When necessary, Tukey post hoc tests were conducted. An $\alpha$ level of .05 was adopted for all statistical tests, which were conducted using SPSS version $22 .^{\mathrm{g}}$

Within-group effect sizes (baseline test and 1 week after the last gait training session; baseline test and 6 weeks after the last training session) were calculated as the difference in mean values from each assessment divided by the pooled SD. Effect sizes were defined using Cohen $d$ classifications $(d=0.2$, small; $d=0.5$, medium; $d=0.8$, large) ${ }^{29}$

\section{Results}

Thirty-two individuals with a mean age of $58.2 \pm 9.1$ years were randomized into the study. Random assignment generated groups that were comparable in terms of age and time poststroke.
Twenty-eight participants completed the training protocol in the allocated group and were included in the final analyses (table 1).

During each set of assessments, 11 participants ( 5 from the overground training group, 6 from the TMG) used assistive devices to perform the 10MWT and $6 \mathrm{MWT}$ and 8 of these participants (4 from each group) used light hand assistance for balance from the same therapist during their gait analyses. All individuals were interested, motivated, and cooperative throughout the training period and assessments, and none of them reported any intervention-related adverse effects. All participants performed all assessments, except the 6MWT, which 6 individuals did not perform ( 3 from the overground training group, 3 from the TMG) because this test was added to the study after they had begun training and after these individuals had self-reported walking longer distances.

Throughout the training period, no differences were observed between the groups for the percentage of BWS (TMG: $16.81 \% \pm$ $7.62 \%$; overground training group: $14.89 \% \pm 6.59 \%$ ) or for session duration (TMG: $37 \pm 6 \mathrm{~min}$; overground training group: $36 \pm 5 \mathrm{~min}$ ). However, the mean comfortable speed set for the treadmill and the servomotor was different (TMG: $.27 \pm .07 \mathrm{~m} / \mathrm{s}$; overground training group: $.52 \pm .07 \mathrm{~m} / \mathrm{s}$ ) despite the fact that the groups were equal during baseline $10 \mathrm{~m}$ walking.

\section{Clinical evaluations}

At 1 week after the last gait training session, both groups demonstrated improvements in gait speed (TMG: $d=.14$; overground training group: $d=.16 ; P=.049$ ), 6MWT (TMG: $d=.35$; overground training group: $d=.30 ; P<.001$ ), FIM (TMG: $d=.41$; overground training group: $d=.27 ; P<.001$ ), and FM (TMG: $d=.82$; overground training group: $d=.55 ; P<.001)$. At 6 weeks after the last training session, both groups maintained these improvements in gait speed (TMG: $d=.25$; overground training group: $d=.32 ; P<.001$ ), 6MWT (TMG: $d=.36$; overground training group: $d=.33 ; P=.001$ ), FIM (TMG: $d=.59$; overground training group: $d=.63 ; P<.001$ ), and FM (TMG: $d=.99$; overground training group: $d=.95 ; P<.001)$. However, there were no

Table 1 General baseline characteristics of individuals

\begin{tabular}{llll}
\hline Characteristic & TMG & OGG & $P$ \\
\hline Sex: female/male & $7 / 7$ & $8 / 6$ & $.71^{*}$ \\
Age (y) & $58.7 \pm 8.4$ & $57.7 \pm 10.1$ & .78 \\
Mass (kg) & $66.7 \pm 11.1$ & $66.0 \pm 12.4$ & .86 \\
Height (m) & $1.63 \pm 0.08$ & $1.60 \pm 0.11$ & .43 \\
Time poststroke (mo) & $60.2 \pm 55.4$ & $53.8 \pm 42.2$ & .73 \\
Type of lesion: I/H & $12 / 2$ & $10 / 4$ & $.37^{*}$ \\
Hemiparesis side: R/L & $5 / 9$ & $8 / 6$ & $.27^{*}$ \\
Mini-Mental State & $24.43 \pm 3.98$ & $21.85 \pm 6.87$ & $.23^{*}$ \\
$\quad$ Examination score & & & \\
Baseline 10MWT (m/s) & $0.69 \pm 0.25$ & $0.73 \pm 0.28$ & .81 \\
Baseline 6MWT (m) & $244 \pm 119$ & $240 \pm 152$ & .94 \\
Baseline FIM score & $80.6 \pm 7.31$ & $83.0 \pm 7.1$ & .46 \\
$\quad$ (maximum, 91) & & & \\
Baseline FM score & $69.2 \pm 6.7$ & $70.9 \pm 8.6$ & .83 \\
$\quad$ (maximum, 84) & & & \\
\hline
\end{tabular}

Abbreviations: $H$, hemorrhagic; I, ischemic; L, left; $0 G G$, overground training group; $\mathrm{R}$, right.

* $\chi^{2}$ test. 
Table 2 Outcome measures of clinical assessments

\begin{tabular}{|c|c|c|c|c|c|c|}
\hline \multirow[b]{2}{*}{ Variable } & \multicolumn{2}{|c|}{ to } & \multicolumn{2}{|c|}{$\mathrm{t} 1$} & \multicolumn{2}{|c|}{ t2 } \\
\hline & Mean \pm SD & $95 \%$ CI & Mean \pm SD & $95 \%$ CI & Mean \pm SD & $95 \%$ CI \\
\hline \multicolumn{7}{|c|}{ 10MWT $(\mathrm{m} / \mathrm{s})$} \\
\hline TMG & $0.66 \pm 0.29$ & $0.50-0.82$ & $0.70 \pm 0.30^{*}$ & $0.52-0.88$ & $0.74 \pm 0.34^{* * *}$ & $0.56-0.93$ \\
\hline OGG & $0.69 \pm 0.30$ & $0.53-0.85$ & $0.74 \pm 0.34^{*}$ & $0.56-0.92$ & $0.79 \pm 0.33^{* * *}$ & $0.60-0.97$ \\
\hline \multicolumn{7}{|c|}{ 6MWT (m) } \\
\hline TMG & $244 \pm 119$ & $158-330$ & $291 \pm 148^{* * *}$ & $201-382$ & $294 \pm 159 * *$ & $199-389$ \\
\hline OGG & $240 \pm 152$ & $154-325$ & $283 \pm 139 * * *$ & $193-373$ & $289 \pm 144^{* *}$ & $193-384$ \\
\hline \multicolumn{7}{|c|}{ FIM score (maximum, 91) } \\
\hline TMG & $80.4 \pm 7.6$ & $76.3-84.4$ & $83.3 \pm 6.6^{* * *}$ & $79.8-86.8$ & $84.5 \pm 6.3^{* * *}$ & $81.5-87.5$ \\
\hline OGG & $82.4 \pm 7.1$ & $78.4-86.5$ & $84.2 \pm 6.1^{* * *}$ & $80.7-87.7$ & $86.1 \pm 4.3^{* * *}$ & $83.1-89.0$ \\
\hline \multicolumn{7}{|c|}{ FM score (maximum, 84) } \\
\hline TMG & $68.7 \pm 7.1$ & $64.3-73.1$ & $74.9 \pm 6.7^{* * *}$ & $70.7-79.0$ & $75.7 \pm 7.0^{* * *}$ & $71.9-79.5$ \\
\hline OGG & $69.4 \pm 8.8$ & $64.9-73.8$ & $74.1 \pm 8.2^{* * *}$ & $70.0-78.3$ & $76.9 \pm 6.8$ & $73.1-80.6$ \\
\hline
\end{tabular}

NOTE. Statistic effect for t0 $\times$ t1 and t0 $\times$ t2; ${ }^{*} P<.05 ;{ }^{*}{ }^{*} P<.01 ;{ }^{* *} P<.001$.

Abbreviations: $\mathrm{CI}$, confidence interval; $\mathrm{OGG}$, overground training group; to, baseline; $\mathrm{t} 1$, posttraining; $\mathrm{t} 2$, follow-up.

differences in improvement between the groups for any of the clinical outcome measures at either 1 week after the last gait training session or 6 weeks after the last training session (table 2).

\section{Gait analyses}

At 1 week after the last gait training session, both groups increased nonparetic step length (TMG: $d=.32$; overground training group: $d=.60 ; P<.001)$; however, only participants from the overground training group increased paretic step length $(d=.32 ; P<.001)$ and improved step length symmetry ratio $(d=1.08 ; P<.001)$, and both groups increased single-limb support duration for the paretic limb only (TMG: $d=.06$; overground training group: $d=.42 ; P=.015)$. At 6 weeks after the last training session, both groups increased step length of both paretic (TMG: $d=.36$; overground training group: $d=.44 ; P<.001)$ and nonparetic (TMG: $d=.40$; overground training group: $d=.56$; $P<.001)$ sides; however, only participants from the overground training group improved step length symmetry ratio $(d=1.08$; $P<.01$ ), and both groups increased single-limb support duration for the paretic limb only (TMG: $d=.14$; overground training group: $d=.79 ; P=.006$ ) (table 3 ).

\section{Discussion}

This study is the first to investigate the effects of time-matched overground versus treadmill gait training with BWS in individuals with stroke. We found that both groups improved their gait speed, endurance, recovery of lower limb motor function impairments, functional independence, nonparetic step length, and single-limb support duration for the paretic limb only immediately after the completion of training and at follow-up. Gait training with overground BWS had an additional benefit of improving step length symmetry ratio compared with treadmill BWS training. In addition, overground BWS training promoted immediate and durable improvement in paretic step length whereas treadmill BWS training improved this outcome measure only at follow-up. Therefore, our hypothesis that overground BWS training would be superior to treadmill BWS training for improvement in walking performance was supported only for step length symmetry ratio.
Gait speed is one of the most common and important measures of functional walking ability in the clinical setting ${ }^{30}$ and is closely associated with functional independence. ${ }^{31,32}$ Both our study groups demonstrated improvements in gait speed immediately after the intervention and at follow-up. The average improvement in gait speed of $.09 \mathrm{~m} / \mathrm{s}$ demonstrated by both training groups would be considered a small to substantial meaningful change in gait speed for individuals with chronic stroke, as defined by Perera et al. ${ }^{33}$ This improvement was similar to or greater than that reported in 2 previous studies ${ }^{5,34}$ of BWS treadmill training that also included a follow-up but was less than that reported in 3 other comparable studies. ${ }^{3,35,36}$ However, all the 3 studies that reported larger improvements used participants who had lower initial walking speeds and therefore may have had the potential to demonstrate greater gains than did our participants. In addition, 2 of these studies ${ }^{35,36}$ used longer training periods. The only previous $\operatorname{study}^{16}$ that investigated the use of overground BWS training reported a similar change in gait speed immediately after training but did not include follow-up testing. Based on the results of the present study, it appears that improvements in gait speed are similar between BWS treadmill training and overground training. Therefore, if a primary goal is to improve gait speed, clinicians should rely on clinical judgment to determine which training method would be most appropriate for an individual patient on the basis of factors such as patient preference, safety, comfort, and other therapeutic goals.

Gains in other clinical measures suggest that our BWS paradigm, in either condition, has benefits other than improving walking speed. Participants in both groups demonstrated a 50-m improvement in 6MWT distance immediately after training and at follow-up. This exceeds the $34.4 \mathrm{~m}$ that has been suggested as the minimal clinically important difference for individuals with stroke. ${ }^{37}$ It is also greater than, or similar to, that reported in other treadmill training studies with and without BWS. Lower extremity impairment, as measured using the FM, also improved by $>7$ points and exceeded the threshold for a perceived meaningful recovery (6 points) by individuals with chronic stroke ${ }^{38}$ and was greater than the 1.5-point improvement demonstrated in the only other BWS training study ${ }^{34}$ reporting FM scores. Finally, the average improvement of 3.9 points in the motor domain of the FIM for our participants was also greater than the 1.7-point 
Table 3 Parameters of gait analyses

\begin{tabular}{|c|c|c|c|c|c|c|}
\hline \multirow[b]{2}{*}{ Variable } & \multicolumn{2}{|c|}{ to } & \multicolumn{2}{|c|}{$\mathrm{t} 1$} & \multicolumn{2}{|c|}{ t2 } \\
\hline & Mean \pm SD & $95 \% \mathrm{CI}$ & Mean \pm SD & $95 \% \mathrm{CI}$ & Mean \pm SD & $95 \% \mathrm{CI}$ \\
\hline \multicolumn{7}{|c|}{ Step length (m) } \\
\hline \multicolumn{7}{|c|}{ TMG } \\
\hline $\mathrm{P}$ & $0.40 \pm 0.11$ & $0.33-0.47$ & $0.42 \pm 0.12$ & $0.35-0.48$ & $0.44 \pm 0.11^{* * *}$ & $0.37-0.51$ \\
\hline NP & $0.35 \pm 0.11$ & $0.28-0.42$ & $0.39 \pm 0.14^{* * *}$ & $0.32-0.45$ & $0.40 \pm 0.14^{* * *}$ & $0.32-0.47$ \\
\hline \multicolumn{7}{|l|}{ OGG } \\
\hline$P$ & $0.39 \pm 0.14$ & $0.32-0.46$ & $0.46 \pm 0.11^{*}$ & $0.39-0.52$ & $0.45 \pm 0.13^{* * *}$ & $0.38-0.52$ \\
\hline NP & $0.37 \pm 0.13$ & $0.31-0.44$ & $0.44 \pm 0.10^{* * *}$ & $0.37-0.51$ & $0.44 \pm 0.12^{* * *}$ & $0.37-0.51$ \\
\hline \multicolumn{7}{|c|}{ Step length symmetry ratio } \\
\hline TMG & $0.80 \pm 0.14$ & $0.73-0.87$ & $0.82 \pm 0.13$ & $0.76-0.87$ & $0.81 \pm 0.13$ & $0.76-0.86$ \\
\hline OGG & $0.84 \pm 0.11$ & $0.77-0.91$ & $0.93 \pm 0.04^{*}$ & $0.87-0.98$ & $0.93 \pm 0.04^{*}$ & $0.88-0.98$ \\
\hline \multicolumn{7}{|c|}{ Single-limb support duration (\%) } \\
\hline \multicolumn{7}{|c|}{ TMG } \\
\hline $\mathrm{P}$ & $25.7 \pm 8.1$ & $21.6-29.6$ & $26.2 \pm 7.6^{*}$ & $22.7-29.8$ & $26.8 \pm 7.2^{* *}$ & $23.2-30.4$ \\
\hline NP & $36.1 \pm 4.6$ & $32.9-39.3$ & $36.0 \pm 4.9$ & $33.0-38.9$ & $36.4 \pm 3.3$ & $33.8-39.0$ \\
\hline \multicolumn{7}{|l|}{ OGG } \\
\hline$P$ & $26.0 \pm 6.33$ & $22.0-30.0$ & $28.4 \pm 4.9^{*}$ & $24.9-31.9$ & $30.8 \pm 5.8^{* *}$ & $27.2-34.5$ \\
\hline NP & $36.9 \pm 6.8$ & $33.8-40.2$ & $37.6 \pm 5.9$ & $34.7-40.6$ & $37.6 \pm 5.9$ & $35.0-40.2$ \\
\hline
\end{tabular}

NOTE. Statistic effect for t0 $\times$ t1 and t0 $\times$ t2; ${ }^{*} P<.05 ;{ }^{* *} P<.01 ;{ }^{* * *} P<.001$.

Abbreviations: $\mathrm{CI}$, confidence interval; NP, nonparetic side; $\mathrm{OGG}$, overground training group; $\mathrm{P}$, paretic side; t0, baseline; $\mathrm{t}$, posttraining; $\mathrm{t}$, follow-up.

change seen in a previous BWS treadmill training study. ${ }^{39}$ In summary, the meaningful improvements in each of the clinical outcome measures support the use of BWS training either on a treadmill or overground, with no differential benefits being observed.

The only outcome measure that was different between the overground training group and the TMG group at follow-up was step length symmetry ratio. This is an important finding for several reasons. First, reduced step length symmetry ratio has been shown to be associated with an increase in fall risk. ${ }^{40}$ Therefore, an improvement in step length symmetry ratio may contribute to a reduction in fall risk during walking. Second, individuals with stroke often desire to look more "normal" 41,42 and having a more symmetrical gait may contribute to that goal especially when accompanied by an increase in speed and endurance. Third, improved symmetry may reduce the energetic cost of walking to increase the functional range or intensity in performance of activities of daily living. ${ }^{43,44}$ Therefore, if a BWS system is available for overground walking, it may be more useful than a treadmillbased system. Although walking on a treadmill by itself will increase symmetry, ${ }^{9}$ this does not appear to carry over well to overground walking, as reported in several studies. ${ }^{5,45}$ Indeed, the overground requirement to intrinsically generate leg movements without extrinsic forcing (eg, the moving treadmill surface) may be an important factor for promoting spatial symmetry.

One caveat to our finding that overground training improved step length symmetry ratio is that the TMG adopted a lower selfselected walking speed for training in comparison to the overground training group. It is typical for individuals to adopt a slower speed on the treadmill than overground, partly because they initially feel less stable on a treadmill, perhaps owing to the lack of visual flow and moving floor surface. ${ }^{8,46}$ Therefore, we cannot be certain that the improvement in step length symmetry ratio was a result of the interaction with a stationary floor surface or from having more repetitions. Because all other variables, including the 10MWT improved similarly across the 2 groups and did not, therefore, show an effect of any increased repetition, we believe that the effect of walking overground in this study is the more likely explanation.

\section{Study limitations}

One limitation of the study was the lack of blinding to treatment by the testers. This could certainly cause bias, although the testers had no specific a priori expectation of which outcome measures were expected to improve differentially between the groups. In addition, the testers were effectively blinded during the gait analysis and could not, therefore, influence these variables including step length symmetry ratio. A second limitation was a lack of a control group, which did not receive any gait training. The main purpose of the present article was the use of partial BWS during gait training either on a treadmill (which is the most common use of BWS for gait interventions) or overground. Because our participants were in the chronic stage, we made the assumption that a control group with no training would be unlikely to show any short-term or follow-up improvements. In addition, most of our participants presented relatively high mental and motor functions, but with some of them using assistive devices or hand assistance for balance during the assessments. This may limit the generalizability to the wider population with stroke. For future studies, we suggest the addition of a control group and inclusion of a greater range of deficit severity with randomization stratified by motor function.

\section{Conclusions}

The use of BWS training either on a treadmill or overground promoted meaningful and durable improvements in gait speed, walking endurance, lower limb function, functional independence, and nonparetic step length. However, only overground training led 
to significant improvements in step length symmetry ratio. Therefore, if BWS gait training is utilized for poststroke gait rehabilitation, it may be useful to include overground training if one of the therapeutic goals is to improve step length symmetry ratio.

\section{Suppliers}
a. Model TK35; CEFISE Biotecnologia Esportiva.
b. BWS system; FENIX Tecnologia.
c. LabVIEW; National Instruments Corp.
d. Model 9286BA; Kistler Instruments Corp.
e. VICON; Oxford Metrics Ltd.
f. MATLAB; The MathWorks Inc.
g. SPSS version 22; IBM Corporation.

\section{Keywords}

Assistive technology; Clinical protocols; Exercise therapy; Rehabilitation

\section{Corresponding author}

Ana M. Barela, PhD, Institute of Physical Activity and Sport Sciences, Cruzeiro do Sul University, Rua Galvão Bueno, 868, São Paulo, SP 01506-000, Brazil. E-mail address: ana.barela@ cruzeirodosul.edu.br.

\section{References}

1. Moseley AM, Stark A, Cameron ID, Pollock A. Treadmill training and body weight support for walking after stroke. Cochrane Database Syst Rev 2005;(4):CD002840.

2. Hesse S, Bertelt C, Jahnke MT, et al. Treadmill training with partial body weight support compared with physiotherapy in nonambulatory hemiparetic patients. Stroke 1995;26:976-81.

3. Sullivan KJ, Knowlton BJ, Dobkin BH. Step training with body weight support: effect of treadmill speed and practice paradigms on poststroke locomotor recovery. Arch Phys Med Rehabil 2002;83:683-91.

4. Hesse S. Treadmill training with partial body weight support after stroke: a review. NeuroRehabilitation 2008;23:55-65.

5. Trueblood PR. Partial body weight treadmill training in persons with chronic stroke. NeuroRehabilitation 2001;16:141-53.

6. Combs-Miller SA, Kalpathi Parameswaran A, Colburn D, et al. Body weight-supported treadmill training vs. overground walking training for persons with chronic stroke: a pilot randomized controlled trial. Clin Rehabil 2014;28:873-84.

7. Manning CD, Pomeroy VM. Effectiveness of treadmill retraining on gait of hemiparetic stroke patients. Physiotherapy 2003;89:337-49.

8. Hesse S, Uhlenbrock D, Sarkodie-Gyan T. Gait pattern of severely disabled hemiparetic subjects on a new controlled gait trainer as compared to assisted treadmill walking with partial body weight support. Clin Rehabil 1999;13:401-10.

9. Harris-Love ML, Forrester LW, Macko RF, Silver KH, Smith GV Hemiparetic gait parameters in overground versus treadmill walking. Neurorehabil Neural Repair 2001;15:105-12.

10. Norman KE, Pepin A, Ladouceur M, Barbeau H. A treadmill apparatus and harness support for evaluation and rehabilitation of gait. Arch Phys Med Rehabil 1995;76:772-8.
11. Brouwer B, Parvataneni K, Olney SJ. A comparison of gait biomechanics and metabolic requirements of overground and treadmill walking in people with stroke. Clin Biomech 2009;24:729-34.

12. Lee SJ, Hidler J. Biomechanics of overground vs. treadmill walking in healthy individuals. J Appl Physiol 2008;104:747-55.

13. Harris-Love ML, Macko RF, Whitall J, Forrester LW. Improved hemiparetic muscle activation in treadmill versus overground walking. Neurorehabil Neural Repair 2004;18:154-60.

14. Kautz SA, Bowden MG, Clark DJ, Neptune RR. Comparison of motor control deficits during treadmill and overground walking poststroke. Neurorehabil Neural Repair 2011;25:756-65.

15. Miller EW, Quinn ME, Seddon PG. Body weight support treadmill and overground ambulation training for two patients with chronic disability secondary to stroke. Phys Ther 2002;82:53-61.

16. Sousa CO, Barela JA, Prado-Medeiros CL, Salvini TF, Barela AM. Gait training with partial body weight support during overground walking for individuals with chronic stroke: a pilot study. J Neuroeng Rehabil 2011;8:48.

17. Barela AMF, Stolf SF, Duarte M. Biomechanics characteristics of adults walking in shallow water and on land. J Electromyogr Kinesiol 2006; 16:250-6

18. Nascimento LR, Caetano LC, Freitas DC, Morais TM, Polese JC, Teixeira-Salmela LF. [Different instructions during the ten-meter walking test determined significant increases in maximum gait speed in individuals with chronic hemiparesis] [Portuguese]. Rev Bras Fisioter 2012;16:122-7.

19. Bohannon RW, Andrews AW, Thomas MW. Walking speed: reference values and correlates for older adults. J Orthop Sports Phys Ther 1996; 24:86-90.

20. Michael K, Goldberg AP, Treuth MS, Beans J, Normandt P, Macko RF. Progressive adaptive physical activity in stroke improves balance, gait, and fitness: preliminary results. Top Stroke Rehabil 2009;16:133-9.

21. ATS Committee on Proficiency Standards for Clinical Pulmonary Function Laboratories. ATS statement: guidelines for the six-minute walk test. Am J Respir Crit Care Med 2002;166:111-7.

22. Riberto M, Miyazaki MH, Jucá SSH, Sakamoto H, Pinto PPN, Battistella LR. Validação da versão brasileira da medida de independência funcional. Acta Fisiátrica 2004;11:72-6.

23. Riberto M, Miyazaki MH, Sakamoto H, Jorge Filho D, Battistella LR. Reprodutibilidade da versão brasileira da medida de independência funcional. Acta Fisiátrica 2000;8:45-52.

24. Maki T, Quagliato EMAB, Cacho EWA, et al. Estudo de confiabilidade da aplicação da escala de Fugl-Meyer no Brasil. Rev Bras Fisioter 2006;10:177-83.

25. Vicon Motion System Limited. Vicon plug-in-gait product guidefoundation notes revision 2.0. Oxford (UK): Vicon Motion System Limited; March 2010.

26. Patterson KK, Gage WH, Brooks D, Black SE, McIlroy WE. Evaluation of gait symmetry after stroke: a comparison of current methods and recommendations for standardization. Gait Posture 2010; 31:241-6.

27. Jiang L, Xu H, Yu C. Brain connectivity plasticity in the motor network after ischemic stroke. Neural Plast 2013;2013:924192.

28. Duncan PW. Stroke disability. Phys Ther 1994;74:399-407.

29. Cohen J. A power primer. Psychol Bull 1992;112:155-9.

30. Dickstein R. Rehabilitation of gait speed after stroke: a critical review of intervention approaches. Neurorehabil Neural Repair 2008; 22:649-60.

31. Schmid A, Duncan PW, Studenski S, et al. Improvements in speedbased gait classifications are meaningful. Stroke 2007;38:2096-100.

32. Perry J, Garrett M, Gronley JK, Mulroy SJ. Classification of walking handicap in the stroke population. Stroke 1995;26:982-9.

33. Perera S, Mody SH, Woodman RC, Studenski SA. Meaningful change and responsiveness in common physical performance measures in older adults. J Am Geriatr Soc 2006;54:743-9.

34. Middleton A, Merlo-Rains A, Peters DM, et al. Body weightsupported treadmill training is no better than overground training for 
individuals with chronic stroke: a randomized controlled trial. Top Stroke Rehabil 2014;21:462-76.

35. Combs SA, Dugan EL, Ozimek EN, Curtis AB. Effects of bodyweight supported treadmill training on kinetic symmetry in persons with chronic stroke. Clin Biomech 2012;27:887-92.

36. Visintin M, Barbeau H, Korner-Bitensky N, Mayo NE. A new approach to retrain gait in stroke patients through body weight support and treadmill stimulation. Stroke 1998;29:1122-8.

37. Tang A, Eng JJ, Rand D. Relationship between perceived and measured changes in walking after stroke. J Neurol Phys Ther 2012;36:115-21.

38. Pandian S, Arya KN, Kumar D. Minimal clinically important difference of the lower-extremity Fugl-Meyer assessment in chronic-stroke. Top Stroke Rehabil 2016;23:233-9.

39. Ribeiro T, Britto H, Oliveira D, Silva E, Galvao E, Lindquist A. Effects of treadmill training with partial body weight support and the proprioceptive neuromuscular facilitation method on hemiparetic gait: a comparative study. Eur J Phys Rehabil Med 2013;49:451-61.

40. Balasubramanian CK, Bowden MG, Neptune RR, Kautz SA. Relationship between step length asymmetry and walking performance in subjects with chronic hemiparesis. Arch Phys Med Rehabil 2007;88:43-9.
41. Hsu AL, Tang PF, Jan MH. Analysis of impairments influencing gait velocity and asymmetry of hemiplegic patients after mild to moderate stroke. Arch Phys Med Rehabil 2003;84:1185-93.

42. Couillandre A, Maton B, Breniere Y. Voluntary toe-walking gait initiation: electromyographical and biomechanical aspects. Exp Brain Res 2002;147:313-21.

43. Nilsson L, Carlsson J, Danielsson A, et al. Walking training of patients with hemiparesis at an early stage after stroke: a comparison of walking training on a treadmill with body weight support and walking training on the ground. Clin Rehabil 2001;15:515-27.

44. Da Cunha IT Jr, Lim PA, Qureshy H, Henson H, Monga T, Protas EJ. Gait outcomes after acute stroke rehabilitation with supported treadmill ambulation training: a randomized controlled pilot study. Arch Phys Med Rehabil 2002;83:1258-65.

45. Combs SA, Dugan EL, Ozimek EN, Curtis AB. Bilateral coordination and gait symmetry after body-weight supported treadmill training for persons with chronic stroke. Clin Biomech 2013;28:448-53.

46. Bayat R, Barbeau H, Lamontagne A. Speed and temporal-distance adaptations during treadmill and overground walking following stroke. Neurorehabil Neural Repair 2005;19:115-24. 